\title{
ESTRUCTURA ATÓMICA: ANÁLISIS Y ESTUDIO DE LAS IDEAS DE LOS ESTUDIANTES (8 DE EGB)
}

\author{
DE LA FUENTE, ANA MARÍA ${ }^{2}$, PERROTTA, MARÍA TERESA², DIMA, GILDA ${ }^{2}$, \\ GUTIÉRREZ, ELENA ${ }^{2}$, CAPUANO, VICENTE ${ }^{1}$, FOLLARI, BEATRIZ ${ }^{2}$ \\ ${ }^{1}$ Facultad de Ciencias Exactas, Físicas y Naturales. Unidad Nacional de Córdoba \\ Vélez Sársfield 1600. 5000 Córdoba \\ vcapuano@com.uncor.edu \\ ${ }^{2}$ Facultad de Ciencias Exactas y Naturales. Univeridad Nacional de La Pampa \\ Uruguay 151. 6300 Santa Rosa (La Pampa) \\ adelafuente@exactas.unlpam.edu.ar
}

Resumen. Este trabajo se propone indagar las ideas de los estudiantes del tercer ciclo de la educación general básica (EGB) en la República Argentina, jóvenes de 12 a 14 años, con el propósito de conocer lo que piensan sobre la estructura atómica. Utilizando una metodología cuantitativa, se implementó una encuesta que se llevó a cabo con 78 alumnos. El cuestionario utilizado, validado en dos oportunidades, fue elaborado por los autores de este trabajo, tomando resultados de entrevistas previas llevadas a cabo en grupos pequeños de perfil similar al de la muestra definitiva y teniendo en cuenta las conclusiones de investigaciones previas del grupo. Los alumnos encuestados habían recibido oportunamente instrucción acerca del átomo y sus constituyentes.

Palabras clave. Estructura de la materia, estructura atómica, concepciones, preadolescentes.

Summary. The purpose of this paper is to find out the pupils' ideas in the Third Cycle of General Basic Education (EGB) in the Argentinean Republic; it was done with young people of between twelve and fourteen years of age, with the aim of knowing what they think about the atomic structure. Using a quantitative methodology, it was carried out interviewing 78 pupils: The questionnaire used, validated in two opportunities, was prepared by the authors of this paper, taking results of previous interviews carried out in small groups of a similar profile to the definitive sample and taking into account the conclusions of previous researches of these groups. The students interviewed had received instruction about the atom and its components.

Keywords. Matter structure, atomic structure, conceptions, preadolescent.

\section{INTRODUCCIÓN}

El trabajo que se presenta es parte de un proyecto $^{1}$ que propone como relevante indagar las ideas de los estudiantes, desde la edad de 12 hasta los 20 años, con el propósito de conocer lo que piensan acerca de un tema de física moderna que figura en los currículos a lo largo del período de la vida escolar que hemos considerado analizar. La elección del tema ha surgido, por un lado, del análisis de los programas de estudio de los distintos niveles educativos que corresponden al rango de edades mencionadas y, por otro, teniendo cuenta resultados de una investigación anterior que muestra con claridad que los alumnos están interesados en los temas que propone la física moderna (Capuano et al., 1997). Dado que estructura atómica se trata en todos los ciclos con diferentes grados de profundidad, es el tema de estudio seleccionado.
La reforma educativa en Argentina de ámbito regional, provincial y nacional, introduce la necesidad de abordar con buen rendimiento, en relación con los aprendizajes de los alumnos, temas del área de la física moderna. También se ha propuesto esto desde el campo de la investigación (Gil Pérez et al., 1986; McDermott, 1993; Capuano et al., 1997).

Según lo expresado por Ostermann y Moreira (2000), quienes resumen las razones respecto a la necesidad de actualizar los currículos de física en la escuela secundaria, coincidimos en que en los currículos actuales se trata la física anterior a 1900 sin hacer mención de las nuevas ideas desarrolladas durante el siglo Xx. Los estudiantes, a través de los distintos medios de comunicación a los que tienen acceso, obtienen información sobre temas como agujeros 
negros, big bang, energía nuclear, etc., de los que nunca oyen hablar en las clases de física. Generalmente se dice que la física moderna es difícil y abstracta, pero, sin embargo, la física clásica presenta dificultades similares y eso no impide que se incluya en los currículos. Si se desea interesar a los alumnos en la ciencia y mostrar una visión más clara y actualizada del trabajo científico deben incluirse temas desarrollados por la física durante el siglo Xx. Así sería posible motivar a los estudiantes para que sean los futuros profesores e investigadores en esta área de la ciencia.

Si bien el avance científico-tecnológico creciente tiene gran sustento en la física desarrollada en el último siglo, en los últimos niveles de la educación general básica (EGB) y en el nivel polimodal (en la República Argentina, ciclo de la enseñanza posterior al EGB para jóvenes de 15 a 17 años) ésta es un área de estudio poco desarrollada, dada la escasez de material bibliográfico con respecto a cómo enfocar los temas (Capuano et al., 1977; Ostermann y Moreira, 2000).

La teoría del aprendizaje de Ausubel plantea como importante, para lograr un aprendizaje significativo, «averiguar lo que el alumno ya sabe y enseñar en consecuencia». Estudios realizados en los últimos veinte años, en relación con el conocimiento de los estudiantes, se han centrado en el ámbito de la educación las ideas de los alumnos sobre un determinado tema, previas al momento de su enseñanza en la escuela. Algunas de las ideas que tienen los alumnos son espontáneas y otras provocadas por la instrucción y suelen causar dificultades para el aprendizaje de nuevos conceptos (Ausubel, 1996).

Es sabido que puede existir más de una idea sobre un mismo fenómeno (Maloney y Siegler, 1993) y que existe una progresiva evolución, en algunos casos, de las concepciones de los estudiantes a lo largo de los años escolares dentro de un área específica del conocimiento (Osborne y Freyberg, 1991), aunque existen diferencias importantes en la evolución y persistencia de ellas en distintos campos (Carrascosa y Gil Pérez, 1987).

Los temas relacionados con las ideas previas sobre la física moderna han sido menos estudiados que en otros capítulos de la física (Pfundt y Duit, 1994) y, en general, no se ha analizado la evolución de dichas ideas en un lapso prolongado de la vida de los estudiantes.

Con referencia a la enseñanza de la estructura de la materia, tema muy ligado a la estructura atómica, se percibe un razonable conjunto de investigaciones multidisciplinarias, que provienen de la enseñanza de la química (Ferro et al., 1995; Furió et al., 1999; Pozo y Gómez Crespo, 1998), de la enseñanza de la física en el ámbito del calor y la temperatura (Cárdenas y Ragout de Lozano, 1996; Domínguez Castiñeiras et al., 1998) y del área que estudia la naturaleza corpuscular o continua de la materia (Benarroch, 2000; Benarroch, 2001; Pozo y Gómez Crespo, 1998). Si bien, en general, no son trabajos estructurados para detectar ideas previas en la estructura cognitiva de los estudiantes, en sus desarrollos y en algún apartado, éstas se hacen presentes.
Hay trabajos de investigación que destacan la importancia de introducir el análisis microscópico a partir de la enseñanza de la física clásica (en calor y temperatura, en electrostática, en electrodinámica, etc.) para poder trabajar luego con lo muy pequeño en la estructura de la materia y la estructura del átomo (Stefanel, 1998). El problema de «cómo explicar lo muy grande y lo muy pequeño», tema que naturalmente involucra la estructura de la materia y la estructura atómica, es también investigado (De la Torre, 1998).

En relación con la enseñanza de la estructura del átomo, también hay trabajos de investigación (Solbes et al., 1987; Gutiérrez et al., 2000) aun cuando es marcadamente menor el número de aportes en la literatura.

En esta primera etapa del trabajo y en razón de que nuestras investigaciones están orientadas a hallar estrategias adecuadas para la enseñanza de la física moderna, indagamos aquí las ideas de los alumnos de $8^{\circ}$ acordando con Lang da Silveira y Moreira (1996), en que esta detección proporcionará información relevante necesaria para intentar una enseñanza que lleve a un aprendizaje significativo de estos temas.

\section{METODOLOGÍA}

En este trabajo se utilizó una metodología cuantitativa a través de un cuestionario, con el propósito de establecer qué idea tienen acerca de la estructura de la materia los niños de 13-14 años. Se encuestaron 78 alumnos de una escuela de la ciudad de Santa Rosa que asistían a $8^{\circ}$ año de EGB, durante el año 2000. El cuestionario fue elaborado por los integrantes del proyecto tomando resultados de entrevistas previas llevadas a cabo en grupos pequeños de perfil similar al de la muestra definitiva (Moreira y Lang da Silveira, 1993) y teniendo en cuenta las conclusiones de investigaciones previas del grupo (Gutiérrez et al., 2000). Fue validado en dos oportunidades hasta llegar a su versión final (Dima et. al., 2000). Los alumnos encuestados habían recibido oportunamente instrucción acerca del átomo y las partículas que lo constituyen

\section{Instrumento de evaluación}

El cuestionario (Anexo I) consta de 14 ítems, de los cuales los primeros 5 ítems indagan sobre si el átomo es la parte más pequeña que constituye las cosas (seres vivos y no vivos) y sobre cómo está constituido. En los ítems 6 al 9 se pregunta sobre la diferencia entre átomos, el movimiento de las partes que lo componen, la masa y el tamaño de las partículas. Las interacciones y las posibilidades de romper o unir el núcleo del átomo se tratan en los ítems 10 a 13. El ítem 14 pretende indagar respecto del origen de la información que tienen los estudiantes.

\section{RESULTADOS Y ANÁLISIS DE DATOS}

En esta sección se consignan los resultados obtenidos en cada pregunta y el análisis de los mismos. Se realizaron 
dos tipos de análisis: uno por cada ítem y otro comparando las respuestas a dos ítems diferentes pero relacionados entre sí. Cabe señalar que todos los porcentajes fueron obtenidos sobre el total de alumnos encuestados.

Pregunta 1: ¿Cómo se construyen internamente los objetos inanimados?

Un $46 \%$ responde «átomo», un $8 \%$ dice «molécula», un $15 \%$, cantidad importante de encuestados, da otras respuestas como: «madera», «tornillos», «árbol», «minerales», etc. Hay un $29 \%$ que no contesta y $1 \%$ que manifiesta «no me acuerdo».

Se observa que cerca de la mitad de los alumnos reconoce como la parte más pequeña al átomo, que es la respuesta esperada por nosotros. Puede interpretarse también como aceptable la respuesta «molécula» que dio otro grupo, dado que la pregunta no es suficientemente específica.

\section{Pregunta 2: ¿Cómo están construidos los seres vivos?}

En este caso, entre los que contestan, la respuesta mayoritaria es «célula» $(35 \%)$, sólo un $15 \%$ responde «átomo» y un 3\%, «molécula». Un 15\% da otras respuestas como «carne», «semilla», «dientes», «raíz». Un 31\% no contesta.

En el caso de los seres vivos, la respuesta más frecuente es «célula» aunque es posible pensar que la interpretación dada por los niños a esta pregunta es «la parte más pequeña que tenga vida», o cuál es la menor unidad funcional. Esto queda evidenciado por los que incluyeron moléculas y átomos dentro de la célula y aún así respondieron «célula». En este caso hubo un porcentaje muy significativo de alumnos que no contesta. Tal vez la pregunta produce algún tipo de desorientación.

\section{Pregunta 3: Diagrama de inclusiones para la gota de agua}

Ningún alumno fue capaz de realizar un esquema correcto completo, ya sea incluyendo o no los quarks. Sin embargo, el $50 \%$ ubicó la molécula dentro de la gota de agua y un $41 \%$, el átomo dentro de la molécula. Sólo un $27 \%$ ubican separados el núcleo y el electrón dentro del átomo, mientras un $10 \%$ incluye el electrón en el núcleo. Un 15\% ubica dentro del átomo el protón, el neutrón, el electrón y el núcleo como cuatro elementos separados. El porcentaje que no contesta es importante (26\%).

En cuanto a los quarks, algunos encuestados aclararon que no los conocían. Un $35 \%$ los ubicó en diferentes posiciones pero no aparece ningún patrón repetitivo, lo que indica que muchos intentaron ubicarlos sólo porque figuraba en la lista de palabras. Un $40 \%$ de los alumnos opta por omitirlos directamente.

Como ninguno fue capaz de realizar un esquema completo se infiere que no tienen claro el concepto de estructura de la materia. Sin embargo, aproximadamente la mitad ubicó correctamente la molécula y el átomo, pero no sus componentes.

\section{Pregunta 4: Diagrama de inclusiones para el perro}

Nuevamente ningún encuestado logró completar la secuencia pero uno de ellos (1\%) hizo un esquema correcto sin incluir los quarks.

Sólo un 19\% logró construir la secuencia perro-corazóncélula. Apenas un $13 \%$ ubicó la molécula dentro de la célula. Un $21 \%$ ubicó el átomo dentro de la molécula pero sólo un 5\% logró incluir correctamente el electrón y el núcleo con el neutrón y el protón en su interior. Un 49\% no contesta y un $1 \%$ declara «no sé». Hay un 5\% que realiza dibujos disparatados.

Para los quarks vale lo comentado en la pregunta 3 aunque en este caso hay un solo alumno que ubicó un quark correctamente dentro del protrón. Un $23 \%$ los pone en cualquier parte y un $26 \%$ los omite.

Del bajo porcentaje que logró reproducir la secuencia perro-corazón-célula se puede ver que no está claro que las células son parte de los órganos. Menos aún son los que incluyen dentro de la célula la molécula y el átomo. Casi la mitad no contesta, lo que completa el cuadro de desorientación con respecto a la estructura de los seres vivos.

\section{Pregunta 5: Dibujo del átomo}

Todos los que intentaron hacer el dibujo representaron el modelo de Bohr. Ninguno mencionó o ubicó los quarks. Un 4\% lo dibujó plano identificando todas las partes y colocando correctamente las cargas. Un 13\% hizo lo mismo en el espacio. Un 33\% realizó gráficos aceptables a los que les faltaba identificar partes o las cargas pero manifestando conocer el modelo. Un $26 \%$ no responde. El resto realiza gráficos incompletos y un $9 \%$ hace dibujos confusos. Todos intentaron dibujar un sistema orbital.

El hecho de que todos los que intentaron el dibujo representaran el modelo de Bohr pone de manifiesto que es lo que se presenta en la instrucción. Aproximadamente la mitad de los alumnos conoce el modelo.

Dado que las cinco primeras cuestiones recogen respuestas que pueden complementarse, se realizó un análisis en forma conjunta de las mismas. De este análisis surge el siguiente resultado:

- Un $15 \%$ contesta «átomo» tanto en el caso de objetos inanimados (pregunta 1) como en los seres vivos (pregunta 2).

- Un 28\% responde «átomo» a la pregunta 1 y «célula» a la pregunta 2 .

- Los que contestan «átomo» en la pregunta 1 y lo ubican correctamente en la gota de agua (pregunta 3 ) son un $23 \%$.

- Un 26\% tiene un esquema aceptable del átomo (pregunta 5) pero no lo ubica adecuadamente (pregunta 3) para los objetos no vivos, mientras un $41 \%$ tiene bien el esquema pero no lo ubica correctamente en el caso de los seres vivos (pregunta 4). 
- Un $8 \%$ dice que la parte más pequeña de los seres vivos es la célula pero en la pregunta 4 incluye dentro de ella la molécula y el átomo en forma correcta.

Al comparar las respuestas dadas a las preguntas 3 y 4 con las correspondientes a la pregunta 5 se puede ver que la idea de átomo que poseen los alumnos está desconectada de sus ideas sobre la materia, ya que muchos de ellos no logran ubicar el átomo dentro de los objetos o de los seres vivos.

Pregunta 6: ¿Qué partes del átomo están quietas y cuáles en movimiento?

\section{Partes quietas}

El 44\% de los alumnos no responde a esta pregunta. Entre los que responden, muchos mencionaron más de una parte, por lo que los porcentajes suman más de 100. El 19\% respondió «núcleo», el 20\% señaló «neutrones» y un 21\%, «protones». Un 20\% menciona «órbitas». Un 14\% da otras respuestas utilizando palabras que figuran en las preguntas 3 y 4 que no corresponden a partes del átomo.

\section{Partes en movimiento}

En este caso, un $45 \%$ no contesta. El $38 \%$ contesta «electrón» pero también son significativos los porcentajes de quienes contestan «protones» $(17 \%)$, «neutrones» $(14 \%)$, las «órbitas» $(12 \%)$, o «el núcleo» $(4 \%)$ o el «átomo» $(5 \%)$. Un $9 \%$ da respuestas extrañas mencionando elementos de las preguntas 3 y 4 .

El alto porcentaje de encuestados que no responde pone en evidencia el hecho de que ésta es una cuestión que tal vez no se han planteado. Entre los que responden aparece tenuemente la idea del núcleo (y sus componentes) en reposo y fuertemente la idea de los electrones en movimiento orbital. Es curioso que un $20 \%$ señale las órbitas en reposo, lo que da la impresión de que se les otorga entidad propia, tal vez como una vía por la que circulan los electrones. Es probable que no puedan representarse otro tipo de movimiento diferente del orbital para los electrones.

Pregunta 7: ¿Hay diferencias entre el átomo de O y el de $\mathrm{Fe}$ ?

Un $65 \%$ responde que son distintos. Entre éstos, un $25 \%$ da una explicación mientras que un $40 \%$ no explica.

Las explicaciones se agruparon en tres categorías:

a) Contestan que son distintos porque la cantidad de protones y electrones son diferentes $(6 \%)$.

b) Dan características de cada uno que los distinguen. Por ejemplo, que el $\mathrm{O}$ es gaseoso, el hierro es un metal, etc. $(13 \%)$.

c) Dan explicaciones incorrectas $(6 \%)$.

Un 13\% contesta que son iguales. Dan explicaciones sólo un $6 \%$, expresando algunos que los dos se oxidan y otros, que todos los átomos son iguales.
Expresó que no sabe un 17\% y no contesta un 5\%.

Si bien los alumnos han tenido instrucción en química sobre la tabla periódica, no comprenden por qué un átomo corresponde a un elemento determinado, aunque la mayoría cree que cada elemento tiene átomos diferentes. Es muy bajo el porcentaje de quienes manifiestan conocer sus diferencias.

Pregunta 8: ¿Cuántos átomos hay aproximadamente en un grano de arena?

La respuesta mayoritaria es «algunos millones» (31\%). El 26\% responde «trillones de átomos». Un 15\% elige «una docena», el 8\% da otras respuestas como «muchísimos», «infinidades». El 14\% responde «no sé» y el $6 \%$ «no contesta».

Seguramente, para los niños, millones o trillones de átomos (respuesta correcta) significa simplemente «muchísimos». Un $65 \%$ elige alguna de estas opciones , por lo que parece que tienen idea de lo pequeño que es un átomo.

Pregunta 9: Relación entre las masas de los constituyentes del átomo

a) Si la masa del protón fuera como la de una naranja, la masa del electrón sería como...

- Un 36\% respondió correctamente «una semilla de naranja». Además, un $6 \%$ indica en «otra respuesta» que es más chiquito o despreciable.

- Un 24\% señaló la opción «una manzana» y un 4\% mas da respuestas equivalentes en «otra respuesta», expresando que las masas de ambos son similares.

- Un 10\% señala como una sandía.

- Un $12 \%$ «no sabe» y 7\% «no contesta». El 1\% no responde a la consigna.

b) Si la masa del protón fuera como la de una naranja la masa del neutrón sería como...

- El 26\% indica «una manzana», a los que se les debe sumar un 3\% de «otra respuesta», que dicen que sería como una naranja.

- El 29\% compara la masa del neutrón con la de una semilla de naranja.

- Un $2 \%$ da otra respuesta comparándola con la de objetos pequeños, tales como una semilla de kiwi.

- Un 13\% indica «como una sandía».

- Un $9 \%$ no contesta. Un $18 \%$ expresa que no sabe.

De la comparación de las masas de las partes del átomo surge que un porcentaje significativo de alumnos tiene claro que el electrón es mucho más pequeño que el protón. En el caso del neutrón, las respuestas correctas disminuyen y hay 
un $31 \%$ que opina que el neutrón es pequeño en relación con el protón. Da la impresión de que la idea de que los electrones son pequeños frente a los protones está más incorporada que la de los neutrones. Tal vez en la instrucción no se los mencione tanto.

Pregunta 10: ¿Por qué se mantienen los electrones alrededor del núcleo?

El $64 \%$ no contesta y un $4 \%$ manifiesta «no sé». Sólo un $5 \%$ responde en forma correcta mencionando la carga negativa del electrón. Un 1\% habla de una fuerza de atracción. Un $10 \%$ da respuestas que apelan a la energía necesaria para mantenerse en órbita o dicen que electrón y protón no podrían estar juntos porque se rechazarían o chocarían. Un $15 \%$ ensaya respuestas incorrectas.

En cuanto a una justificación de por qué los electrones se mantienen en órbita (según el modelo que los niños conocen), está claro que los alumnos no están en condiciones de darla. Es muy importante el porcentaje que no contesta y apenas un $6 \%$ da respuestas aproximadas. En este caso debe tenerse en cuenta que los alumnos desconocen tanto las leyes de Newton y su aplicación al movimiento circular como la electrostática, por lo que no tienen claro que existe una atracción entre protón y electrón.

Pregunta 11: ¿Se pueden separar los electrones de un átomo?

La respuesta mayoritaria es «no» (41\%), de éstos el 17\% no explica por qué, el resto trata de dar una explicación. Las ideas más frecuentes son:

- El átomo no puede perder los electrones porque dejaría de ser un átomo.

- El átomo no se puede dividir porque es muy pequeño.

Un 33\% contestó que se pueden separar, respuesta esperada. De éstos un $21 \%$ no explica por qué. El resto ensaya explicaciones aunque ninguna correcta. El 3\% da una explicación aproximada, del tipo «porque los electrones son los que se mueven». El 10\% restante da explicaciones incorrectas o del tipo «porque creo que es así»».

El 17\% no sabe y el $9 \%$ no contesta.

Entre los alumnos que contestaron, la mayoría cree que no se pueden separar los electrones del átomo. En un grupo está presente la idea de que, si se separan los electrones, «dejamos de tener un átomo».

Probablemente no se les ha mencionado nada sobre la ionización. Además, aunque se les hable de electrización por frotamiento, tal vez no se les haya dicho que los electrones son los que pasan de un cuerpo a otro.

\section{Pregunta 12: ¿Se puede partir el núcleo del átomo?}

El $55 \%$ señala que no se puede partir. Un $29 \%$ de ellos no explica por qué. El $26 \%$ restante da alguna explicación; la más frecuente es que el átomo es indivisible y no es posi- ble romperlo, pues dejaría de ser un átomo. Otros indican que es demasiado pequeño para poder dividirlo.

Un $21 \%$ contesta que sí. Sólo un $8 \%$ da una explicación, de los cuales el $3 \%$ dice que se separa por un choque y el $5 \%$ restante da explicaciones no satisfactorias.

Un $15 \%$ contesta que no sabe y un $9 \%$ no contesta.

En cuanto a la posibilidad de partir el núcleo, la mayoría cree que no se puede. Inclusive los que dicen que sí, no dan una explicación apropiada. Al parecer, en la instrucción nunca se menciona nada referido a la fisión. Por el contrario, está muy arraigado el concepto de que el átomo es indivisible (palabra utilizada por los niños).

Pregunta 13: ¿Se pueden unir dos núcleos para formar un núcleo más grande?

Entre los que contestan, la respuesta mayoritaria es «no» (33\%). De éstos un $18 \%$ no explica. El $15 \%$ restante expresa ideas tales como que el «átomo es indivisible y no se puede juntar», son muy pequeños y «no se pueden ver ni reformar».

La respuesta afirmativa (esperada) logró un 28\%, de los cuales el $18 \%$ no explica. Sólo uno responde que se pueden unir y da el nombre correcto del fenómeno: «fusión nuclear». Un 3\% piensa que, si son pequeños, se pueden juntar los protones para hacer un núcleo más grande con mayor cantidad. El resto da explicaciones no satisfactorias.

Un $28 \%$ manifiesta que no sabe y un $10 \%$ no contesta.

La mayoría dice que no pueden unirse dos núcleos para formar uno más grande, por lo que da la impresión de que en la instrucción no se menciona la fusión. El hecho de que un solo niño respondiera correctamente hace pensar que este conocimiento es extraescolar.

Pregunta 14: Origen de la información con la que cuentan los alumnos

Un $82 \%$ indica que la adquirieron en la escuela, mientras un $14 \%$ contesta «en televisión», un $4 \%$ en diarios y en revistas un $11 \%$. Un 7\% dice que la información les llegó a través de charlas con amigos o familiares. Un $17 \%$ no contesta. Cabe aclarar que la suma de los porcentajes es mayor que 100 porque los encuestados podían optar por varias posibilidades.

En la tabla I se presenta un resumen de los resultados de las respuestas de los alumnos al cuestionario.

\section{CONCLUSIONES}

En general los alumnos no tienen ideas claras acerca de la estructura de la materia, ya que no han podido realizar la secuencia inclusiva propuesta en el cuestionario, lo que revela que, si bien se conoce el átomo, no se reconoce como 
Tabla I

Registro de las respuestas dadas por los alumnos y su porcentaje.

\begin{tabular}{|c|c|c|}
\hline PREGUNTAS & Porcentaje (\%) & RESPUESTAS \\
\hline $\begin{array}{l}\text { 1. ¿Cómo se construyen internamente los objetos } \\
\text { inanimados? }\end{array}$ & $\begin{array}{r}46 \\
8 \\
- \\
15 \\
30\end{array}$ & $\begin{array}{l}\text { Átomo } \\
\text { Molécula } \\
\text { Partículas subatómicas (quarks) } \\
\text { Tornillo, madera, minerales } \\
\text { No contesta }\end{array}$ \\
\hline 2. ¿Cómo se construyen internamente los seres vivos? & $\begin{array}{r}15 \\
35 \\
3 \\
- \\
15 \\
31\end{array}$ & $\begin{array}{l}\text { Átomo } \\
\text { Célula } \\
\text { Molécula } \\
\text { Quarks } \\
\text { Carne, semilla, raíz, diente } \\
\text { No contesta }\end{array}$ \\
\hline 3. Diagrama de inclusiones para la gota de agua & $\begin{array}{r}- \\
50 \\
41 \\
- \\
15 \\
27 \\
35 \\
40 \\
26\end{array}$ & $\begin{array}{l}\text { Esquema correcto incluyendo o no los quarks } \\
\text { Ubica la molécula dentro de la gota de agua } \\
\text { Ubica el átomo dentro de la molécula } \\
\text { Ubica correctamente núcleo, } e^{-}, p^{+} \text {y } n \\
\text { Ubica separados núcleo, } e^{-}, p^{+} \text {y } n \\
\text { Ubica separados núcleo y } e^{-} \text {dentro del átomo } \\
\text { Ubica mal los quarks } \\
\text { Omite los quarks } \\
\text { No contesta }\end{array}$ \\
\hline 4. Diagrama de inclusiones para el perro & $\begin{array}{r}- \\
19 \\
13 \\
21 \\
5 \\
23 \\
26 \\
49\end{array}$ & $\begin{array}{l}\text { Esquema correcto incluyendo o no los quarks } \\
\text { Secuencia correcta perro-corazón-célula } \\
\text { Ubica la molécula dentro de la célula } \\
\text { Ubica al átomo dentro de la molécula } \\
\text { Ubica correctamente núcleo, } e^{-}, p^{+} \text {y } n \\
\text { Ubica mal los quarks } \\
\text { Omite los quarks } \\
\text { No contesta }\end{array}$ \\
\hline 5. Dibujo del átomo & $\begin{array}{r}4 \\
13 \\
33 \\
26\end{array}$ & $\begin{array}{l}\text { Modelo de Bohr (órbitas en un plano) } \\
\text { Modelo de Bohr (órbitas en el espacio) } \\
\text { Modelo de Bohr algo incompleto } \\
\text { No contesta }\end{array}$ \\
\hline $\begin{array}{l}\text { 6. ¿Qué partes del átomo están quietas y cuáles en } \\
\text { movimiento? }\end{array}$ & $\begin{array}{r}19 \\
20 \\
21 \\
20 \\
44 \\
\\
- \\
38 \\
17 \\
14 \\
12 \\
45\end{array}$ & $\begin{array}{l}\text { Quietas } \\
\text { Núcleo } \\
\text { Neutrones } \\
\text { Protones } \\
\text { Órbitas } \\
\text { No contesta } \\
\text { En movimiento } \\
\text { Todas } \\
\text { Electrones } \\
\text { Protones } \\
\text { Neutrones } \\
\text { Órbitas } \\
\text { No contesta }\end{array}$ \\
\hline $\begin{array}{l}\text { 7. ¿Hay diferencias entre el átomo de oxígeno y el de } \\
\text { hierro? }\end{array}$ & $\begin{array}{r}65 \\
13 \\
17 \\
5\end{array}$ & $\begin{array}{l}\text { Distintos } \\
\text { Iguales } \\
\text { No sé } \\
\text { No contesta }\end{array}$ \\
\hline $\begin{array}{l}\text { 8. ¿Cuántos átomos hay aproximadamente en un grano } \\
\text { de arena? }\end{array}$ & $\begin{array}{r}31 \\
26 \\
15 \\
8 \\
14 \\
6\end{array}$ & $\begin{array}{l}\text { Algunos millones } \\
\text { Algunos trillones } \\
\text { Docenas } \\
\text { Infinidades, muchísimos } \\
\text { No sé } \\
\text { No contesta }\end{array}$ \\
\hline $\begin{array}{l}\text { 9. ¿Qué relación existe entre las masas de los elementos } \\
\text { del átomo? }\end{array}$ & $\begin{array}{r}36+6 \\
24+4 \\
10 \\
12 \\
7\end{array}$ & $\begin{array}{l}\text { Sobre la relación entre el protón y el electrón } \\
\text { Semilla de naranja + chiquita o despreciable } \\
\text { Manzana + naranja } \\
\text { Sandía } \\
\text { No sé } \\
\text { No contesta }\end{array}$ \\
\hline
\end{tabular}




\begin{tabular}{|c|c|c|}
\hline & $\begin{array}{r}23+3 \\
29 \\
13 \\
18 \\
9\end{array}$ & $\begin{array}{l}\text { Sobre la relación entre el protón y el neutrón } \\
\text { Manzana + naranja } \\
\text { Semilla de naranja } \\
\text { Sandía } \\
\text { No sé } \\
\text { No contesta }\end{array}$ \\
\hline $\begin{array}{l}\text { 10. ¿Por qué se mantienen los electrones girando alrededor } \\
\text { del núcleo? }\end{array}$ & $\begin{array}{r}5 \\
1 \\
10 \\
15 \\
4 \\
64\end{array}$ & $\begin{array}{l}\text { Fuerza de atracción entre cargas eléctricas } \\
\text { Fuerza de atracción (explicación incompleta) } \\
\text { Relaciona con la energía } \\
\text { Repulsión, repulsión y atracción, gravedad } \\
\text { No sé } \\
\text { No contesta }\end{array}$ \\
\hline 11. ¿Se pueden separar los electrones de un átomo? & $\begin{array}{r}33 \\
41 \\
17 \\
9 \\
\end{array}$ & $\begin{array}{l}\text { Sí } \\
\text { No } \\
\text { No sé } \\
\text { No contesta }\end{array}$ \\
\hline $\begin{array}{l}\text { 12. ¿Se puede partir el núcleo de un átomo en dos núcleos } \\
\text { más pequeños? }\end{array}$ & $\begin{array}{r}21 \\
55 \\
15 \\
9\end{array}$ & $\begin{array}{l}\text { Sí } \\
\text { No } \\
\text { No sé } \\
\text { No contesta }\end{array}$ \\
\hline $\begin{array}{l}\text { 13. ¿Se pueden unir dos núcleos para formar un núcleo } \\
\text { más grande? }\end{array}$ & $\begin{array}{l}28 \\
33 \\
28 \\
10\end{array}$ & $\begin{array}{l}\text { Sí } \\
\text { No } \\
\text { No sé } \\
\text { No contesta }\end{array}$ \\
\hline $\begin{array}{l}\text { 14. ¿Cuál es el origen de la información que le permitió } \\
\text { responder las preguntas anteriores? }\end{array}$ & $\begin{array}{r}82 \\
11 \\
4 \\
14 \\
7 \\
17\end{array}$ & $\begin{array}{l}\text { En la escuela } \\
\text { Revistas } \\
\text { Diarios } \\
\text { Televisión } \\
\text { Charlas con amigos y familiares } \\
\text { No contesta }\end{array}$ \\
\hline
\end{tabular}

parte de la misma. Varios autores coinciden en que a esta edad los alumnos no poseen una estructura cognitiva en la cual tengan sentido los conceptos de la teoría atómica, y sus respuestas confirmarían que han aprendido memorísticamente (Llorens Molina, 1988; Enciso et al., 1987; Sanz et al., 1993). Probablemente, por tratarse de aspectos muy alejados de los directamente perceptibles por sus sentidos, cuyas dimensiones no les son familiares, los estudiantes no lo entienden (De la Torre, 1998).

Si bien se reconoce como parte más pequeña de la materia el átomo, existe una fuerte diferenciación en las respuestas según se refieran a objetos inanimados (mayoritariamente responden átomo) o a los seres vivos (mayoritariamente responden células), aun cuando un porcentaje discreto de estos últimos reconoce la existencia del átomo (Mondelo Alonso et al., 1994).

El modelo de átomo que al parecer tiene la mayoría de los alumnos de 13 años es un modelo orbital con el núcleo en reposo (tenue tendencia) y los electrones girando a su alrededor aunque no saben por qué, indivisible y muy pequeño. En esto se coincide con la imagen de las partículas como bolas microscópicas (Gil Pérez et al., 1989; Ostermann et al., 2000) o la concepción del átomo como un pequeño sistema planetario (Lahore, 1990). Además, con respecto a la indivisibilidad, muestra la relación con las ideas mantenidas por la comunidad científica a lo largo de la historia.

No han podido explicar lo que diferencia un átomo de otro, como tampoco según el modelo que ellos conocen por qué los electrones se mantienen unidos al núcleo. Mayoritariamente piensan que los electrones no se separan del átomo y si esto ocurriera dejaría de existir el mismo. Son menores los conocimientos que tienen los alumnos al indagar sobre el núcleo en particular, tanto respecto a la fisión como a la fusión.

En lo que respecta a los quarks, se advierte su desconocimiento, lo que señalaría que en la instrucción no está incorporado este tema.

Los alumnos en su mayoría manifiestan que las respuestas dadas al cuestionario presentado provienen de la instrucción recibida en la escuela. Muy lejos aparece la influencia de la televisión, diarios y revistas. Incluso no resulta relevante la influencia del medio (amigos y familiares). Este cuadro de situación indica claramente que las ideas sobre estructura atómica que se aceptan científicamente, no se han socializado, y que la falta de estímulo que provoca su ausencia en la naturaleza visible llevan a olvidar lo que se aprende en la escuela. Los alumnos no tienen experiencias previas en estos temas y tanto en la estructura 
de la materia como en la estructura del átomo se tratan aspectos muy alejados de los directamente perceptibles por los sentidos de los jóvenes (Solbes, 1996; De la Torre,1998).

De las tres vertientes de enfoques metodológicos que mencionan Ostermann y Moreira (2000), y teniendo en cuenta lo señalado por los alumnos, en relación a que las respuestas dadas al cuestionario presentado provienen de la instrucción recibida en la escuela, se puede inferir que la enseñanza de la física moderna se llevaría a cabo adhiriendo a la primera: presentación de los sistemas clásicos para luego enfatizar sobre el carácter no lineal del desarrollo científico, las dificultades que generaron las crisis de la física clásica y las profundas diferencias conceptuales entre la física clásica y moderna (Gil et al., 1989). Naturalmente, sólo estamos en condiciones de afirmar que en el caso de la estructura atómica, la presentación del tema se realiza utilizando un sistema clásico aun cuando no poseemos información sobre el enfoque de dicha presentación.

Cada vez que se trata la problemática de enseñar la física del siglo xx, se señala que la dificultad se asienta en que las nuevas teorías son muy complicadas, en la compleja herramienta matemática necesaria para abordarlas, en la necesidad de utilizar modelos que resultan antiintuitivos, en las dificultades (propias del costo y complejidad de los equipos) para llevar a cabo experiencias de laboratorio, en las serias dificultades (que se pueden discutir) para encontrar aplicaciones de los conceptos de física desarrollados en la cotidianeidad y, finalmente, en la enorme cantidad de conocimientos previos que se necesita para poder comprender los modelos de la física del siglo xx (Fernández et al., 1997). Esta reflexión está indicando que uno de los inconvenientes más importantes en la enseñanza de la física del siglo $\mathrm{xx}$ en general, y en el de la estructura atómica, en particular, es el buen manejo de las ideas que proporciona la totalidad de la física clásica (previa al siglo Xx). Los jóvenes encuestados apenas si habían recibido un par de cursos elementales de física y de química; en consecuencia, no sólo no habían sido entrenados en el trabajo de «lo muy pequeño» (Stefanel, 1998; De la Torre, 1998), sino que no disponían de un buen manejo de las ideas que proporciona la totalidad de la física clásica.

Lo expuesto motiva a este grupo de investigación a analizar en trabajos posteriores el rol que juega la instrucción planteada por el sistema educativo en la evolución de estas ideas y a favorecer el planteo de estrategias que tiendan a mejorar el proceso de enseñanza-aprendizaje de este tema.

\section{NOTA}

${ }^{1}$ Análisis y estudio de la evolución de las concepciones de los estudiantes en algunos temas de física moderna. Aprobado por la Facultad de Ciencias Exactas y Naturales de la Universidad Nacional de La Pampa. La dirección de este proyecto está a cargo de Vicente Capuano, de la Facultad de Ciencias Exactas, Físicas y Naturales de la Universidad Nacional de Córdoba.

\section{REFERENCIAS BIBLIOGRÁFICAS}

AUSUBEL, D., NOVAK L. y HANESIAN, H. (1996). Psicología educativa. Un punto de vista cognoscitivo. México: Trillas.

BENARROCH, A. (2000). El desarrollo cognoscitivo de los estudiantes en el área de la naturaleza corpuscular de la materia. Enseñanza de las Ciencias, 18(2), pp. 235-246.

BENARROCH, A. (2001). Una interpretación del desarrollo cognoscitivo de los alumnos en el área de la naturaleza corpuscular de la materia. Enseñanza de las Ciencias, 19(1), pp. 123-134.

CAPUANO, V., GUTIÉRREZ, E., PERROTTA, M., DE LA FUENTE, A. y FOLLARI, B. (1997). Física moderna: ausente en la escuela media (tercer ciclo de EGB y nivel polimodal), Memoria REF X, tomo 2, 2c-03, Mar del Plata, Argentina.
CÁRDENAS, M. y RAGOUT DE LOZANO, S. (1996). Explicaciones de procesos termodinámicos a partir del modelo corpuscular: una propuesta didáctica. Enseñanza de las Ciencias, 14(3), pp. 343-349.

CARRASCOSA, J. y GIL, D. (1987). Diferencias en la evolución de preconceptos de mecánica y química. Actas del II Congreso Internacional de Investigación en Didáctica de las Ciencias y Matemática. Enseñanza de las Ciencias. Número extra, p. 167.

DE LA TORRE, A.C. (1998). Cómo explicar lo grande y lo pequeño. Revista de Enseñanza de la Física (APFA), 11(1), pp. 53-56.

DIMA G., CAPUANO V., PERROTTA, M., DE LA FUENTE A., FOLLARI, B. y GUTIÉRREZ, E. (2000). Elaboración y validación de un cuestionario sobre algunos temas de física 
moderna. Memorias de V Simposio de Investigación en Educación en Física. Publicado en CD: «Metodología de la Investigación Científica».

DOMÍNGUEZ CASTIÑEIRAS, J. M., DE PRO BUENO, A. y GARCÍA-RODEJA FERNÁNDEZ, E. (1998). Las partículas de la materia y su utilización en el campo conceptual de calor y temperatura: un estudio transversal. Enseñanza de las Ciencias, 16(3), pp. 461-475.

ENCISO, E., LLORENS, J.A. y SENDRA, F. (1987). La introducción al modelo corpuscular de la materia. Enseñanza de las Ciencias, núm. extra, pp. 183-184.

FERNÁNDEZ, P.E., GONZÁLEZ, E. y SOLBES, J., (1997). Algo más que una ampliación de contenidos. Revista de Educación en Ciencias de la Universidad Nacional de Gral. San Martín, 1(3), pp. 5-10.

FERRO, V. R., GONZÁLEZ-JONTE, R. H. y CRUZ, Z. (1995). Una reflexión curricular sobre la enseñanza de la estructura de la sustancia en la formación de profesores de química. Enseñanza de las Ciencias, 13(3), pp. 371-377.

FURIÓ, C., AZCONA, R. y GUISASOLA, J. (1999). Dificultades conceptuales y epistemológicas del profesorado en la enseñanza de los conceptos de cantidad de sustancia y de mol. Enseñanza de las Ciencias, 17(3), pp. 359-376.

GIL PÉREZ, D., SENENT, F. y SOLBES, J. (1986). Análisis crítico de la introducción de la física moderna en la enseñanza media. Revista de Enseñanza de la Física, 2(1), pp. 16-21.

GIL PÉREZ, D., SENENT, F. y SOLBES, J. (1989). Física moderna en la enseñanza secundaria: una propuesta fundamentada y sus resultados. Revista Española de Física, 3, pp. 53-58.

GUTIÉRREZ, E., CAPUANO, V., PERROTTA, M., DE LA FUENTE, A. y FOLLARI, B. (2000). ¿Qué piensan los jóvenes sobre radioactividad, estructura atómica y energía nuclear? Enseñanza de las Ciencias, 18(2), pp. 247-254.

LAHORE, A. (1990). Una encuesta sobre el concepto de átomo en los estudiantes. Enseñanza de las Ciencias 8(3), p. 307.

LANG DA SILVEIRA, F. y MOREIRA, M.A. (1996). Validación de un test para verificar si el alumno posee concepciones científicas sobre calor, temperatura y energía interna. Enseñanza de las Ciencias, 14(1) pp. 75-86.

LLORENS MOLINA, J.A. (1988). Propuesta y aplicación de una metodología para el análisis de la adquisición de conceptos en la introducción a la teoría atómica molecular: percepción de los hechos experimentales, sus representaciones y el uso del lenguaje en alumnos de formación profesional y bachillerato. Enseñanza de las Ciencias, 6(2), pp. 200-202.

MALONEY, D. y SIEGLER, R. (1993). Conceptual competition in physics learning. International Journal of Science Education, 15(3), pp. 283-295.

McDERMOTT, L. (1993). Cómo enseñamos y cómo aprenden los estudiantes. ¿Un desajuste? Primera y segunda parte. Enseñanza de la Física, 6(1-2), pp. 19-32, 19-28.

MONDELO ALONSO, M., GARCÍA BARROS, S. y MARTÍNEZ LOSADA, C. (1994). Materia inerte / materia viva ¿Tienen ambas constitución atómica? Enseñanza de las Ciencias, 12(2), pp. 226-233.

MOREIRA, M.A. y LANG DA SILVEIRA, F. (1993). Instrumento De Pesquisa Em Ensino \& Aprendizagem. Porto Alegre, Brasil: Edipucrs.

OSBORNE, R. y FREYBERG, P. (1991). El aprendizaje de las ciencias. Implicaciones de la ciencia de los alumnos. Madrid: Narcea.

OSTERMANN, F. y MOREIRA, M.A. (2000). Física contemporánea en la escuela secundaria: una experiencia en el aula involucrando formación de profesores. Enseñanza de las Ciencias, 18(3), pp. 391-404.

POZO, J.I. y GÓMEZ CRESPO, M.A. (1998). Aprender y enseñar ciencia. Madrid: Ediciones Morata.

PFUNDT, H. y DUIT, R. (1994).Bibliography Student's alternative frame works and Science Education (4a. ed.). Kiel, Germany: IPN.

SANZ, A. GÓMEZ CRESPO, M.A. y POZO, J.I. (1993). Influencia de la instrucción en la utilización del modelo de partículas. Enseñanza de las Ciencias, núm. extra (IV Congreso), pp. 281-282.

SOLBES, J. (1996). Física moderna y su enseñanza. Alambique, 10 , pp. 59-67.

SOLBES, J., CALATAYUD, M., CLIMENT, J. y NAVARRO, J. (1987). Errores conceptuales en los modelos atómicos cuánticos. Enseñanza de las Ciencias, 5(3), pp. 189-195.

STEFANEL, A. (1998). Una experiencia en el marco de la introducción de la física cuántica en la escuela secundaria. Revista de Enseñanza de la Física (APFA), 11(2), pp. 35-44. 


\section{ANEXO I}

Año que cursa.

Unidad educativa núm.

Las siguientes cuestiones intentan conocer tus ideas acerca de las partes más pequeñas que forman la materia. Responde cada una de las preguntas o señala con una cruz la/s opción/es que consideres correcta/s.

1) Nombra cuál es la parte más pequeña con la que se construyen internamente :

una mesa / una piedra / una hoja de papel / el aire / el agua

Respuesta:

2) Nombra cuál es la parte más pequeña con la que se construyen internamente:

un perro / un rosal / un pez / un árbol / una mariposa

Respuesta:

3) Con las siguientes palabras:

departamento / cama / sala / dormitorio / edificio / cocina / barrio / mesa de luz

se confeccionó un diagrama que muestra que el edificio está dentro del barrio, que el departamento está dentro del edificio, que el dormitorio, la sala y la cocina están dentro del departamento, etc.

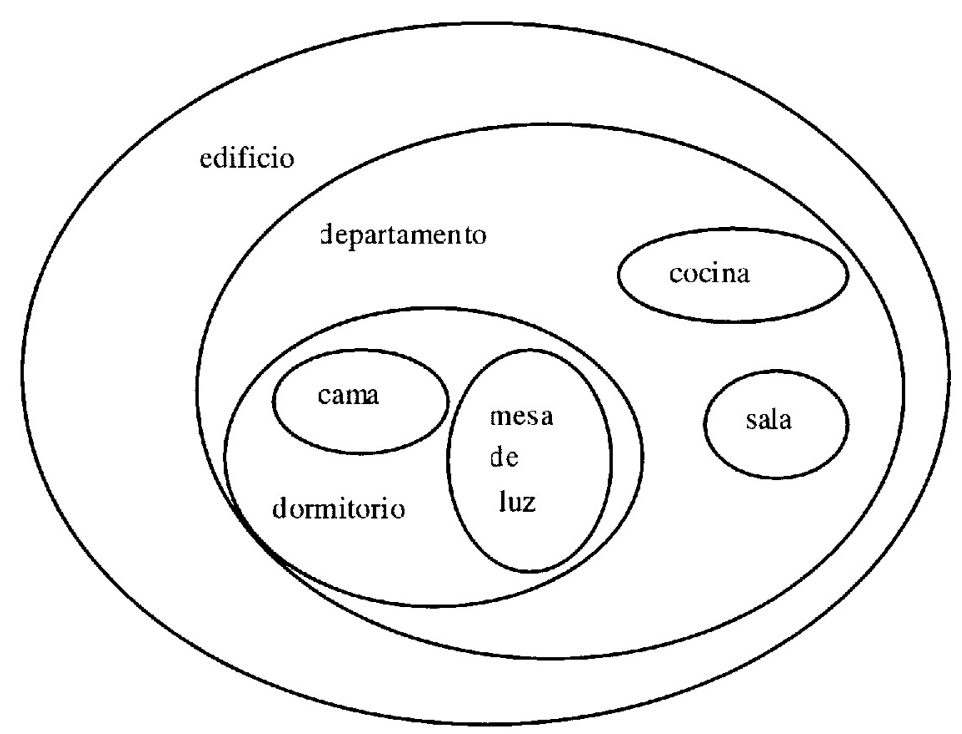

Confecciona un diagrama similar al anterior para el caso de una gota de agua con las siguientes palabras átomo / neutrón / núcleo / protón / molécula / quarks / electrón / gota de agua

Respuesta: 


\section{INVESTIGACIÓN DIDÁCTICA}

4) Confecciona un diagrama similar a los anteriores, para el caso de un perro con las siguientes palabras:

átomo / neutrón / célula / núcleo / protón / molécula / quarks / corazón/ electrón / perro

Respuesta

5) Realiza un dibujo que represente un átomo y nombra cada una de sus partes.

Respuesta

6) Indica qué partes del dibujo anterior consideras que están quietas y qué partes están en movimiento:

Partes quietas

Partes en movimiento.

7) Un átomo de hierro y un átomo de oxígeno son:

$$
\begin{aligned}
& \square \text { iguales } \\
& \square \text { distintos } \\
& \square \text { no sé }
\end{aligned}
$$

Explica por qué elegiste esa respuesta.

8) ¿Cuántos átomos crees, aproximadamente, que hay en un grano de arena?

$$
\begin{aligned}
& \square \text { una docena de átomos } \\
& \square \text { algunos millones } \\
& \square \text { trillones de átomos } \\
& \square \text { otra respuesta:..................... } \\
& \square \text { no sé }
\end{aligned}
$$

9) Si la masa del protón fuera como la de una naranja

- La masa del electrón sería como la de:

$$
\begin{aligned}
& \square \text { una semilla de naranja } \\
& \square \text { una manzana } \\
& \square \text { una sandía } \\
& \square \text { otra respuesta:................... } \\
& \square \text { no sé }
\end{aligned}
$$

- La masa del neutrón sería como la de:

$$
\begin{aligned}
& \square \text { una semilla de naranja } \\
& \square \text { una manzana } \\
& \square \text { una sandía } \\
& \square \text { otra respuesta:................... } \\
& \square \text { no sé }
\end{aligned}
$$

10) Los electrones se mantienen alrededor del núcleo formando el átomo. Explica por qué crees que ocurre esto.

Respuesta 


\section{INVESTIGACIÓN DIDÁCTICA}

11) ¿Se pueden separar los electrones de un átomo?

$\square$ sí

$\square$ no

$\square$ no sé

Explica por qué elegiste esa respuesta.

12) ¿Se puede partir el núcleo del átomo?

$\square$ sí

$\square$ no

$\square$ no sé

Explica por qué elegiste esa respuesta.

13) ¿Se pueden unir dos núcleos pequeños para formar un núcleo más grande?
$\square$ sí
$\square$ no
$\square$ no sé

Explica por qué elegiste esa respuesta.

14) ¿Cuál es el origen de esta información que nos brindaste?
$\square$ La televisión
$\square$ La escuela
$\square$ Revistas
$\square$ Diarios
$\square$ Charlas con amigos o familiares 